\title{
A fixed point method to solve differential equation and Fred- holm integral equation
}

\author{
Ei Ei Nyein, Aung Khaing Zaw* \\ School of Mathematics and Statistics, Beijing Institute of Technology, Beijing 100081, China.
}

\begin{abstract}
The purpose of this research is to explore a fixed point method to solve a class of functional equations, $T u=f$, where $T$ is a differential or an integral operator on a Sobolev space $H^{2}(\Omega)$, where $\Omega$ is an open set in $\mathbb{R}^{n}$. First, T is converted into a sum of $\mathrm{I}+\lambda A$ with $\lambda>0$, where $A$ is a continuous linear operator and $I$ is identity mapping. Then it is shown that $T$ is a contraction on the prescribed Sobolev space and norm of $A$ is estimated on the prescribed Sobolev space. By means of the theory of inverse operator of $I+\lambda A$ and by choosing the appropriate value of $\lambda$, the solution $u$ of differential or integral operator is obtained. Some practical problems concerning the linear differential equation and Fredholm integral equation are solved by virtue of the fixed point method.
\end{abstract}

Keywords: Fixed point method, ODE and PDE, Fredholm integral equation, estimation.

2010 MSC: 47H10, 45B05.

(C)2020 All rights reserved.

\section{Introduction}

Mathematical aspects of differential and integral equations with extensive applications have obtained a lot of consideration in various research areas, and the theory of differential and integral equations is also arising with basic mathematical tools such as fixed point theory, topology and functional analysis. There are several ways to solve differential equation by inverse differential operator [2, 4-13]. This paper derived a new method of fixed point to functional equation by means of norm operator $A$ on the Sobolev space $H^{m}(\Omega)$ by [1]. Fixed point methods in order to solve functional equation like $T u=f$ where $T$ may be a differential and integral operator have been adapted by Browder [3] and Kangtunyakarn [12]. They created new notions and concept to handle functional equations especially based on monotone operator theory, fixed point theory, linear operator theory, and variational inequalities. There are a large number of generalizations for this interesting theorem, for example see [2, 7, 9, 11]. And then Kakde [11] by using the fixed point theory existence and uniqueness of solution on differential and integral equation, see also Kragler [13] who studied the method of inverse differential operator which is well established for

\footnotetext{
*Corresponding author

Email addresses: eieinyein1985@yahoo.com (Ei Ei Nyein), akzbee451986@yahoo.com (Aung Khaing Zaw)
}

doi: 10.22436/jnsa.013.04.05 
ordinary differential equations can be applied to certain class of partial differential equation. This paper is one of the results due to them and we have an improved estimate of derivatives and integrals by using differentiation of distributions and norm of functions on Sobolev space.

Here, we organized three parts in this study. The first part concerns estimation of norm of linear operator on the prescribed Sobolev space. The second part involves solving linear differential equations by means of fixed point method. The third regards solving integral equation of Fredholm type. Existence and Uniqueness of fixed point is guaranteed by Banach fixed point principle and finding the fixed point is the required solution of the prescribed functional equation.

\section{Preliminaries}

Definition 2.1 ([1]). Let $\mathrm{H}^{\mathrm{m}}(\Omega)=\left\{\phi \in \mathrm{L}^{2}(\Omega) \mid \mathrm{D}^{\alpha} \phi \in \mathrm{L}^{2}(\Omega)\right.$ for all $\left.\alpha:|\alpha| \leqslant \mathrm{m}\right\}$, where $\alpha=\left(\alpha_{1}, \alpha_{2}, \ldots, \alpha_{n}\right) \in$

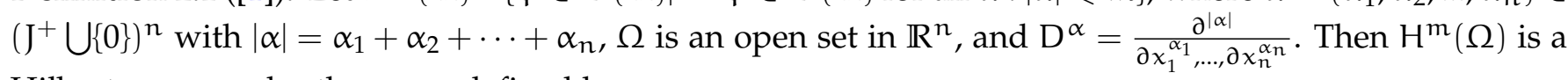
Hilbert space under the norm defined by

$$
\|\phi\|_{m, 2}=\left(\sum_{|\alpha| \leqslant m}\left\|D^{\alpha} \phi\right\|_{\mathrm{L}^{2}}^{2}\right)^{\frac{1}{2}}
$$

By the definition, $\|\phi\|_{\mathrm{m}, 2}^{2}=\left(\sum_{|\alpha| \leqslant m}\left\|\mathrm{D}^{\alpha} \phi\right\|_{\mathrm{L}^{2}}^{2}\right)$ and hence

$$
\|\phi\|_{m, 2} \geqq\left\|D^{\alpha} \phi\right\|_{L^{2}, \forall \alpha:|\alpha| \leqslant m} .
$$

Theorem 2.2. Let $\phi \in \mathrm{H}^{\mathrm{m}}(\Omega), \mathrm{u} \in \mathrm{D}^{\prime}(\Omega)$.Then $\left\|\mathrm{D}^{\alpha}\right\| \leqq 1, \forall \alpha:|\alpha| \leqslant \mathrm{m}$.

Proof. By differentiation of distribution, we have

$$
\left\langle D^{\alpha} u, \phi\right\rangle=(-1)^{|\alpha|}\left\langle u, D^{\alpha} \phi\right\rangle \text {. }
$$

Then $\mid\left\langle\mathrm{D}^{\alpha} \mathrm{u}, \phi\right\rangle \leqq\|\mathrm{u}\|\left\|\mathrm{D}^{\alpha} \phi\right\|, \forall \phi \in \mathrm{H}^{\mathrm{m}}(\Omega)$. So,

$$
\left\langle\mathrm{D}^{\alpha} \mathrm{u}, \phi\right\rangle \leqq\|u\|\|\phi\|_{\mathrm{m}, 2} \text {. }
$$

Therefore, we have $\left\|D^{\alpha} u\right\| \leqq\|u\|, \forall u \in D^{\prime}(\Omega)$. Since $D^{\alpha}$ is linear. $\left\|D^{\alpha}\right\| \leqq 1, \forall \alpha:|\alpha| \leqq m$. In particular, $\left\|\frac{\mathrm{d}}{\mathrm{dx}}\right\| \leqq 1$ and $\left\|\frac{\mathrm{d}^{2}}{\mathrm{~d} x^{2}}\right\| \leqq 1$.

Theorem 2.3. Let $A$ be a continuous linear operator defined on $\mathrm{H}^{\mathrm{m}}(\Omega)$ and $\lambda>0, f \in \mathrm{H}^{\mathrm{m}}(\Omega)$. Suppose the operator $\mathrm{T}$ defined by $\mathrm{Tu}=\lambda \mathrm{Au}+\mathrm{f}$. Then $\mathrm{T}$ is a contraction if $|\lambda|\|A\|<1$.

Proof. For any $u, v \in \mathrm{H}^{\mathrm{m}}(\Omega)$,

$$
\|\mathrm{Tu}-\mathrm{T} v\|=\|\lambda A u-\lambda A v\| \leqslant|\lambda|\|A\|\|u-v\| .
$$

Since $|\lambda|\|A\|<1, T$ is a contraction.

Theorem 2.4. Let $\mathrm{T}$ be as in Theorem 2.3. Then $\mathrm{Tu}=\mathrm{u}$ if and only if $(\mathrm{I}-\lambda \mathrm{A}) \mathrm{u}=\mathrm{f}, \forall \mathrm{u} \in \mathrm{H}^{\mathrm{m}}(\Omega)$.

Proof. Let $\mathrm{Tu}=\mathfrak{u}$. Then

$$
\lambda A u+f=u .
$$

So,

$$
I u-\lambda A u=f, \quad(I-\lambda A) u=f .
$$

Its converse is clear. By the result in Theorem 2.3, the fixed point $u$ of $T$ is a solution of the functional equation $(I-\lambda A) u=f$. Moreover, since $|\lambda|\|A\|<1$ is invertible and also

$$
u=(I-\lambda A)^{-1} f=f+\lambda A f+\lambda^{2} A^{2} f+\cdots+\lambda^{n} A^{n} f+\cdots=\sum_{n=0}^{\infty} \lambda^{n} A^{n} f
$$

where $A^{0}=I$. 


\section{Application to ordinary differential equation}

\subsection{Solution of ordinary differential equation}

Consider $a \frac{d^{2} u}{d x^{2}}+b \frac{d u}{d x}+c u=f, c \neq 0$, then by Theorem 2.2, we have obtained

$$
\left\|\frac{\mathrm{d}}{\mathrm{d} x}\right\| \leqq 1 \text { and }\left\|\frac{\mathrm{d}^{2}}{\mathrm{~d} x^{2}}\right\| \leqq 1
$$

So,

$$
\left\|\mathrm{a} \frac{\mathrm{d}^{2}}{\mathrm{~d} x^{2}}\right\| \leqq|\mathrm{a}| \text { and } \quad\left\|\mathrm{b} \frac{\mathrm{d}}{\mathrm{dx}}\right\| \leqq|\mathrm{b}|
$$

and hence

$$
\left\|\mathrm{a} \frac{\mathrm{d}^{2}}{\mathrm{~d} x^{2}}+\mathrm{b} \frac{\mathrm{d}}{\mathrm{dx}}\right\| \leqq 2 \max \{|\mathrm{a}|,|\mathrm{b}|\}
$$

Let $A u=-\frac{a}{c} \frac{d^{2} u}{d x^{2}}-\frac{b}{c} \frac{d u}{d x}$, define the operator $T$ by

$$
\mathrm{Tu}=\mathrm{Au}+\frac{\mathrm{f}}{\mathrm{c}} .
$$

Since,

$$
a \frac{d^{2} u}{d x^{2}}+b \frac{d u}{d x}+c u=f, \quad-\frac{a}{c} \frac{d^{2} u}{d x^{2}}-\frac{b}{c} \frac{d u}{d x}=u-\frac{f}{c}, \quad A u+\frac{f}{c}=u,
$$

(or)

$$
\mathrm{Tu}=\mathrm{u} .
$$

So, we obtain the following theorem.

Theorem 3.1. Let $\mathrm{Bu}=\mathrm{a} \frac{\mathrm{d}^{2} \mathrm{u}}{\mathrm{d} x^{2}}+\mathrm{b} \frac{\mathrm{du}}{\mathrm{dx}}+\mathrm{cu}=\mathrm{f}, \mathrm{c} \neq 0, \max \{|\mathrm{a}|,|\mathrm{b}|\}<\frac{1}{2}|c|$. Then $\mathrm{Bu}=\mathrm{f}$ is equivalent to $\mathrm{Tu}=\mathrm{u}$ and hence $\mathrm{Bu}=\mathrm{f}$ has a unique solution $\mathrm{u}$.

Proof. Let $\mathrm{Tu}=\mathrm{A} u+\frac{\mathrm{f}}{\mathrm{c}}$ where $\mathrm{A}$ is prescribed above.

$$
\|A\|=\left\|-\frac{a}{c} \frac{d^{2}}{d x^{2}}-\frac{b}{c} \frac{d}{d x}\right\| \leqq\left|\frac{a}{c}\right|+\left|\frac{b}{c}\right| \leqq \frac{2}{|c|} \max \{|a|,|b|\}<\frac{1}{|c|} \cdot|c|=1,
$$

for $\max \{|a|,|b|\}<\frac{1}{2}|c|$. Then $\|T u-T v\|=\|A u-A v\| \leqq\|A\|\|u-v\|$. Since $\|A\|<1, T$ is a contraction on $H^{2}(\Omega)$, there is a unique fixed point $u \in H^{2}(\Omega)$ by Banach fixed point theorem. Therefore, $u$ is a unique solution of $\mathrm{Tu}=\mathrm{f}$ and

$$
u=\frac{1}{c}(I-A)^{-1} f=\frac{1}{c}\left(f+A^{2} f+\cdots+A^{n} f+\cdots \square\right.
$$

Example 3.2. Consider $a \frac{d^{2} u}{d x^{2}}+b \frac{d u}{d x}+c u=f$, where $a=0.1, b=0.3, c=4$, and $f(x)=x^{2}$. Then $2 \max \left\{\left|\frac{\mathrm{a}}{\mathrm{c}}\right|,\left|\frac{\mathrm{b}}{\mathrm{c}}\right|\right\}=\frac{0.3}{2}=0.15<1$. So, we obtain the solution

$$
\begin{aligned}
u=\frac{1}{c}(I-A)^{-1} f=\frac{1}{c}\left[I+A+A^{2}+\cdots\right] f & =\frac{1}{c}\left[f+A f+A^{2} f+\cdots\right] \\
& =\frac{1}{4}\left(x^{2}+\left(-\frac{1}{20}-\frac{3}{20} x\right)+\frac{9}{800}\right) \\
& \left.=\frac{1}{4}\left(x^{2}-\frac{1}{20}-\frac{3}{20} x\right)+\frac{9}{800}\right)=\frac{1}{4} \chi^{2}-\frac{3}{80} x-\frac{31}{3200} .
\end{aligned}
$$


When $\|A\|=1$, we may still use the $(I+A)^{-1}$ method, provide the equation is stable, i.e., $u_{n}$ is a solution of the equation $T_{n} u=f, T_{n} \rightarrow T$ and $u_{n} \rightarrow u_{\infty}$ implies that $u_{\infty}$ is a solution of $T u=f$. In that case, when $\|A\|=1$, we may consider the equation $\left(I+A_{n}\right) u=f$ where $A_{n}=\frac{n}{(n+1)} A$. Then $\|A\|<1$ and $A n \rightarrow A$. Suppose $u_{n}$ is a solution of $u+A_{n} u=f, u_{n} \rightarrow u_{\infty}$. Then $u_{\infty}$ is a solution of $u+A u=f$.

As a particular problem, consider $\frac{d^{2} u}{d x^{2}}+2 u(x)=x-x^{3}+x^{4}$ in $H^{1}(a, b)$. Now $\left\|\frac{d^{2}}{d x^{2}}\right\| \leqq 1$. Hence we consider $\frac{n}{(n+1)} \frac{d^{2} u}{d x^{2}}+2 u(x)=x-x^{3}+x^{4}$ in stead of the original equation. In the new equation,

$$
\left\|\frac{n}{(n+1)} \frac{d^{2}}{d x^{2}}\right\| \leqq \frac{n}{(n+1)}<1
$$

Hence we use $\left(I+A_{n}\right)^{-1}$, where $A_{n}=\frac{n}{(n+1)} \frac{d^{2}}{d x^{2}}$. Here,

$$
\begin{aligned}
u_{n}(x) & =\left(I+\frac{n}{(n+1)} \frac{d^{2}}{d x^{2}}\right)^{-1}\left(x-x^{3}+x^{4}\right) \\
& =\left(I-\frac{n}{(n+1)} \frac{d^{2}}{d x^{2}}+\frac{n^{2}}{((n+1))^{2}} \frac{d^{4}}{d x^{4}}+\cdots\right)\left(x-x^{3}+x^{4}\right), \\
u_{n}(x) & =x-x^{3}+x^{4}+\frac{n}{(n+1)}\left(-6 x+12 x^{2}\right)+\frac{n^{2}}{(n+1)^{2}}(24) .
\end{aligned}
$$

Since $\lim _{n \rightarrow \infty} u_{n}(x)=u(x), u(x)=12+\frac{5}{2} x-6 x^{2}-\frac{1}{2} x^{3}+\frac{1}{2} x^{4}$ is a solution of given equation.

Example 3.3. Let us consider another equation. We know $\left\|\frac{\mathrm{d}}{\mathrm{dx}}\right\| \leqq 1$ but cannot yet establish that it is less than 1 . So we cannot use $(I+A)^{-1}$ method directly. If we use it directly, we obtain by $y=\left(I+\frac{d}{d x}\right)^{-1} \sin x$, $y=\sin x+\cos x-\sin x-\cos x+\sin x+\cos x-\sin x-\cos x+\cdots$, which does not converge. We may consider $\frac{n}{(n+1)} y^{\prime}(x)+2 y(x)=\sin x$. Since $\left\|\frac{n}{(n+1)} \frac{d}{d x}\right\| \leqq \frac{n}{(n+1)}<1$, we have

$$
\begin{aligned}
y_{n}(x) & =\left(I+\frac{n}{(n+1)} \frac{d}{d x}\right)^{-1} \sin x \\
& =\left[I-\frac{n}{(n+1)} \frac{d}{d x}+\frac{n^{2}}{(n+1)^{2}} \frac{d^{2}}{d x^{2}}-\frac{n^{3}}{(n+1)^{3}} \frac{d^{3}}{d x^{3}}+\cdots\right] \sin x \\
& =\sin x\left\{1-\frac{n^{2}}{(n+1)^{2}}+\frac{n^{4}}{(n+1)^{4}}+\cdots\right\}-\cos x\left\{\frac{n}{(n+1)}-\frac{n^{3}}{(n+1)^{3}}+\frac{n^{5}}{(n+1)^{5}}+\cdots\right\} \\
& =\frac{\sin x}{1+\left(\frac{n}{(n+1)}\right)^{2}}-\frac{\frac{n}{(n+1)}}{1+\left(\frac{n}{(n+1)}\right)^{2}} \cos x .
\end{aligned}
$$

$\lim _{n \rightarrow \infty} y_{n}(x)=\frac{1}{4} \sin x-\frac{1}{4} \cos x$, which is a solution of the given equation we considered.

Next, we consider the partial differential operator $T$ defined by

$$
T u=\left(a_{00}+a_{10} \frac{\partial}{\partial x_{1}}+a_{01} \frac{\partial}{\partial x_{2}}+a_{11} \frac{\partial^{2}}{\partial x_{1} \cdot \partial x_{2}}+a_{20} \frac{\partial^{2}}{\partial x_{1}^{2}}+a_{02} \frac{\partial^{2}}{\partial x_{2}^{2}}\right) u=f, a_{00} \neq 0 .
$$

This may be written in the form

$$
\left(I+\frac{a_{10}}{a_{00}} \frac{\partial}{\partial x_{1}}+\frac{a_{01}}{a_{00}} \frac{\partial}{\partial x_{2}}+\frac{a_{11}}{a_{00}} \frac{\partial^{2}}{\partial x_{1} \cdot \partial x_{2}}+\frac{a_{20}}{a_{00}} \frac{\partial^{2}}{\partial x_{1}^{2}}+\frac{a_{02}}{a_{00}} \frac{\partial^{2}}{\partial x_{2}^{2}}\right) u=\frac{f}{a_{00}} .
$$

Let $A=I+\frac{a_{10}}{a_{00}} \frac{\partial}{\partial x_{1}}+\frac{a_{01}}{a_{00}} \frac{\partial}{\partial x_{2}}+\frac{a_{11}}{a_{00}} \frac{\partial^{2}}{\partial x_{1} \cdot \partial x_{2}}+\frac{a_{20}}{a_{00}} \frac{\partial^{2}}{\partial x_{1}^{2}}+\frac{a_{02}}{a_{00}} \frac{\partial^{2}}{\partial x_{2}^{2}}$, under the condition $\|A\|<1$, we obtain

$$
u=\frac{1}{a_{00}}(I+A)^{-1} f=\frac{1}{a_{00}}\left(I-A+A^{2}-A^{3}+\cdots\right) f=\frac{1}{a_{00}} \sum_{n=0}^{\infty}(-1)^{n} A^{n} f,
$$


where $A^{0}=I$. In particular, consider $k \Delta u+u=f$ with $|k|<\frac{1}{2}, \Delta u=\frac{\partial^{2} u}{\partial x_{1}^{2}}+\frac{\partial^{2} u}{\partial x_{2}^{2}}, u \in H^{1}(\Omega)$ and $f \in H^{1}(\Omega)$, $\Omega \subset \mathbb{R}^{2}$ is open. Here, $T=I+k \Delta u$ and $\|k \Delta\|<1$. Then

$$
u=(I+k \Delta)^{-1} f=f-k \Delta f+k^{2} \Delta^{2} f-k^{3} \Delta^{3} f+\cdots=\sum_{n=0}^{\infty}(-1)^{n} k^{n} \Delta^{n} f,
$$

where $\Delta^{0} \mathrm{f}=\mathrm{f}$.

Example 3.4. Consider $\frac{1}{3} \Delta u+u(x)=x_{1}^{3} x_{2}, x=\left(x_{1}, x_{2}\right) \in \mathbb{R}^{2}$. Then

$$
u(x)=\left(I+\frac{1}{2} \Delta\right)^{-1}\left(x_{1}^{3} x_{2}\right)=x_{1}^{3} x_{2}-2 x_{1} x_{2}+4 x_{1}^{2} x_{2}^{2} .
$$

\section{Application to Fredholm integral equations}

\subsection{Fredholm integral equation}

Consider $u(x)+\lambda \int_{a}^{b} K(x, y) u(y) d y=f(x)$, where $(K u)(x)=\int_{a}^{b} K(x, y) u(y) d y$. Then this integral equation can be written as the following functional equation

$$
u+\lambda K u=f, \quad u=f-\lambda K u .
$$

Let $B u=f-\lambda K u$. Then $\|B u-B v\|=\|\lambda K(u-v)\| \leqq|\lambda|\|K\|\|u-v\|$ for $|\lambda|\|K\|<1$, B is a contraction and fixed point of $B$ gives a unique solution of $T u=f$ where $T$ is defined by $T u=u+\lambda K u$.

Since the solution of this integral equation depends on our knowledge of operator $K$, we shall now state a few results about $\mathrm{K}$ which will be used $\|\mathrm{K}\|$ in respective function spaces as follows.

Proposition 4.1 ([8]).

(1) Let $\mathrm{X}=\mathrm{C}([\mathrm{a}, \mathrm{b}])$ for $-\infty<\mathrm{a}<\mathrm{b}<\infty$. Suppose $\mathrm{m}=\sup _{\mathrm{x} \in[\mathrm{a}, \mathrm{b}]} \int_{\mathrm{a}}^{\mathrm{b}} \mathrm{K}(\mathrm{x}, \mathrm{y}) \mathrm{d} \mathrm{y}<\infty$, then $\mathrm{K} \in \mathrm{L}(\mathrm{X}, \mathrm{X})$ and $\|\mathrm{K}\| \leqq \mathrm{m}$.

(2) Let $\mathrm{X}=\mathrm{L}([\mathrm{a}, \mathrm{b}])$ for $-\infty<\mathrm{a}<\mathrm{b}<\infty$. Suppose $\mathrm{n}=\int_{\mathrm{a}}^{\mathrm{b}} \sup _{\mathrm{x} \in[\mathrm{a}, \mathrm{b}]}|\mathrm{K}(\mathrm{x}, \mathrm{y})| \mathrm{d} \mathrm{y}<\infty$, then $\mathrm{K} \in \mathrm{L}(\mathrm{X}, \mathrm{X})$ and $\|K\| \leqq n$.

(3) Let $1<p, p^{1}, q, q^{1}<\infty$ with $\frac{1}{p}+\frac{1}{p^{1}}=\frac{1}{q}+\frac{1}{q^{1}}=1$. Let $r=\max \left\{p^{1}, q\right\}$ and $\frac{1}{r}+\frac{1}{r^{1}}=1$. Suppose $M=$ $\int_{a}^{b} \int_{a}^{b}|K(x, y)|^{r} d x d y<\infty$. Then $K \in L_{(}\left(L^{p}([a, b])\right),\left(L^{q}([a, b])\right.$, and $\|K\| \leqq N M^{\frac{1}{r}}$, where $N=(b-a)^{\alpha+\beta}$, $\alpha=\frac{p-r^{1}}{p r^{1}}, \beta=\frac{r-q}{r q}$. Obviously, if $p=q=r=r^{1}=2$, then we have $\int_{a}^{b} \int_{a}^{b}|K(x, y)|^{2} d x d y<\infty$, $\mathrm{K} \in \mathrm{L}\left(\mathrm{L}^{2}([\mathrm{a}, \mathrm{b}]), \mathrm{L}^{2}([\mathrm{a}, \mathrm{b}])\right)$ with $\|\mathrm{K}\| \leqq\left(\int_{\mathrm{a}}^{\mathrm{b}} \int_{\mathrm{a}}^{\mathrm{b}}|\mathrm{K}(\mathrm{x}, \mathrm{y})| \mathrm{d} x \mathrm{~d} y\right)^{\frac{1}{2}}$. Since $\alpha=\frac{2-2}{2 \times 2}, \beta=\frac{2-2}{2 \times 2}$, and $\mathrm{N}=$ $(b-a)^{0}+0=1$.

(4) Let $\mathrm{X}=\mathrm{L}^{\infty}([\mathrm{a}, \mathrm{b}])$ for $-\infty<\mathrm{a}<\mathrm{b}<\infty$. Suppose $\mathrm{m}=\int_{\mathrm{a}}^{\mathrm{b}} \int_{\mathrm{a}}^{\mathrm{b}}|\mathrm{K}(\mathrm{x}, \mathrm{y})|^{2} \mathrm{~d} x \mathrm{~d} y$, then $\mathrm{K} \in \mathrm{L}(\mathrm{X}, \mathrm{Y})$ and $\|\mathrm{K}\| \leqq \mathrm{m}$ for $\mathrm{Y}=\mathrm{L}^{\prime}([\mathrm{a}, \mathrm{b}])$.

Example 4.2. Consider the integral equation $u(x)+\lambda \int_{0}^{1}(3 x+2 y)^{2} u(y) d y=2-x^{2}$. We shall evaluate the range of $\lambda$ for the fixed point method is applicable in the spaces $C([0,1]), L^{1}([0,1]), L^{2}([0,1])$, and $L^{\infty}([0,1])$.

(i) When we consider in $C([0,1])$,

$$
\|\lambda K\|=|\lambda| \int_{0}^{1} \sup _{x \in[0,1]}(3 x+2 y)^{2} d x=\frac{49}{3}|\lambda| .
$$

Then $\|\lambda K\|<1$ if $|\lambda|<\frac{3}{49}=0.06122$.

(ii) $\operatorname{In~} \mathrm{L}^{1}([0,1])$,

$$
\|\lambda K\| \leqq\left|\lambda \| \int_{0}^{1} \sup _{y \in[0,1]}(3 x+2 y)^{2} d x\right|=13|\lambda| .
$$

Then $\|\lambda K\|<1$ if $|\lambda|<\frac{1}{13}=0.0769$. 
(iii) $\operatorname{In~} \mathrm{L}^{2}([0,1])$,

$$
\|\lambda \mathrm{K}\| \leqq\left|\lambda \|\left(\int_{0}^{1} \int_{0}^{1}|3 x+2 y|^{4} \mathrm{~d} x \mathrm{dy}\right)\right|=\sqrt{82.4}|\lambda|
$$

Then $\|\lambda \mathrm{K}\|<1$ if $|\lambda|<\frac{1}{\sqrt{82.4}}=0.1101$.

(iv) $\operatorname{In} L^{\infty}([0,1])$,

$$
\|\lambda K\| \leqq \sup _{x \in[0,1]} \int_{0}^{1}(3 x+2 y)^{2} d y=\frac{49}{3}|\lambda| .
$$

Then $\|\lambda \mathrm{K}\|<1$ if $|\lambda|<\frac{3}{49}=0.06122$.

Next, we consider the integral equation $(I+\lambda K) u=f$, where $K \in L(X, X), X=L^{2}([0,1])$. If $|\lambda|\|K\|<1$, then $(I+\lambda K)^{-1}$ exists and hence $u=(I+\lambda K)^{-1} f=\sum_{n=0}^{\infty}(-1) \lambda^{n}\left(K^{n} f\right)$ is a solution.

Example 4.3. Consider $u(x)+\frac{1}{10} \int_{0}^{1}(2 x+3 y)^{2} u(y) d y=5 x+1$. Now $\|K\| \leqq \sqrt{82.4}$ and then $|\lambda|\|K\|<1$.

$$
\begin{aligned}
(K f)(x) & =\int_{0}^{1}(2 x+3 y)^{2}(5 y+1) d y=14 x^{2}+26 x+14.25 \\
\left(K^{2} f\right)(x) & =\int_{0}^{1}(2 x+3 y)^{2}\left(14 y^{2}+26 y+14.25\right) d y=127.67 x^{2}+231.5 x+126.45 \\
\left(K^{3} f\right)(x) & =\int_{0}^{1}(2 x+3 y)^{2}\left(127.67 y^{2}+231.5 y+126.45\right) d y=1139.022 x^{2}+2067.71 x+1130.031 \\
& \vdots \\
u(x) & =-1.515 x^{2}+2.181 x-0.546+\cdots
\end{aligned}
$$

Example 4.4. Consider $u(x)+\frac{1}{2} \int_{0}^{1}(x+y) u(y) d y=2-x^{2}$. Now $\|K\|<\sqrt{\frac{7}{6}}$ and hence $\|\lambda K\|<1$. Then $u=(I+\lambda K)^{-1} f$

$$
\begin{aligned}
(K f)(x) & =\int_{0}^{1}(x+y)\left(2-y^{2}\right) d y=1.667 x+0.75 \\
\left(K^{2} f\right)(x) & =\int_{0}^{1}(x+y)\left(2-(1.667 y+0.75)^{2}\right) d y=-0.73875 x-0.8095 \\
\left(K^{3} f\right)(x) & =\int_{0}^{1}(x+y)\left(2-(1.5835 y+0.93066)^{2}\right) d y=0.9286 x+0.1369 \\
& \vdots \\
u(x) & =0.46-1.3275 x-x^{2}+\cdots
\end{aligned}
$$

The convergence of this series has been guaranteed by the Banach fixed point theorem since the operator is a contraction and space $\mathbb{R}$ is a complete metric space.

\section{Conclusion}

A way to solve functional equation has been presented by using upper bounds of norm of operators $T$ which may be a linear differential or a linear partial differential or a Fredholm integral operator on an open subset of Hilbert Sobolev space by transforming $T=I+\lambda A$ where $\|A\|<1$. The inverse operator method has been used in order to obtain required solution of $T u=f$. By using the fixed point theorem, we obtain the existence and uniqueness solution from the results of the prescribed functional equations. 


\section{Acknowledgment}

The author is very grateful to the referee for his/her valuable suggestions and comments to improve the quality of this work.

\section{References}

[1] R. A. Adam, Sobolev Spaces, Academic Press, New York-London, (1975). 1, 2.1

[2] M. A. Alghamdi1, W. A. Kirk, N. Shahzad, Metric fixed point theory for nonexpansive mappings defined on unbounded sets, Fixed Point Theory Appl., 2014 (2014), 12 pages. 1

[3] F. E. Browder, The Solvability of Nonlinear Functional Equations, Duke Math. J., 30 (1963), 557-566. 1

[4] C. Chidume, Iteration Methods for Approximation of Solutions of Nonlinear Equations in Banach Spaces, Ph.D. Thesis (Auburn University), Ann Arbor, (2008). 1

[5] J. B. Conway, A Course in Functional Analysis, Springer-Verlag, New York, (1990).

[6] M. M. El-Borai, W. G. El-Sayed, N. N. Khalefa, Solvability of Some Nonlinear Integral Functional Equations, Amer. J. Theor. Appl. Stat., 6 (2017), 13-22.

[7] A. P. Farajzadeh, A. Kaewcharoen, S. Plubtieng, An Application of Fixed Point Theory to a Nonlinear Differential Equation, Abstr. Appl. Anal., 2014 (2014), 7 pages. 1

[8] S. Goldberg, Unbounded linear operators, McGraw-Hill Book Co., New York, (1966). 4.1

[9] H. H. G. Hashem, A. M. A. El-Sayed, Solvability of Nonlinear Quadratic Functional Equations, J. Appl. Math. Inf. Sci., 9 (2015), 2715-2720. 1

[10] P. Junlouchai, A. Kaewcharoen, S. Plubtieng, A Generalized System of Nonlinear Variational Inequalities in Banach Spaces, Abstr. Appl. Anal., 2014 (2014), 10 pages.

[11] R. V. Kakde, S. S. Biradar, S. S. Hiremath, Solution of Differential and Integral Equations Using Fixed Point Theory, Int. J. Adv. Res. Comput. Eng. Tech. (IJARCET), 3 (2014), 4 pages. 1

[12] A. Kangtunyakarn, Fixed point theory for nonlinear mappings in Banach spaces and applications, Fixed Point Theory Appl., 2014 (2014), 16 pages. 1

[13] R. Kragler, The Method of Inverse Differential Operators Applied for the Solution of PDEs, in: Differential Equations, Dynamical Systems and Celestial Mechanics, 2011 (2011), 79-95. 1 